Théologiques

Théologiques

\title{
Transposition de la morale des prophètes dans la trilogie de Michel Tournier
}

\section{Nancy Saad}

Volume 24, numéro 1, 2016

(In)justice sociale

URI : https://id.erudit.org/iderudit/1044741ar

DOI : https://doi.org/10.7202/1044741ar

Aller au sommaire du numéro

\section{Éditeur(s)}

Faculté de théologie et de sciences des religions, Université de Montréal

ISSN

1188-7109 (imprimé)

1492-1413 (numérique)

Découvrir la revue

Citer cet article

Saad, N. (2016). Transposition de la morale des prophètes dans la trilogie de Michel Tournier. Théologiques, 24(1), 109-130.

https://doi.org/10.7202/1044741ar
Résumé de l'article

La présence de la Bible dans l'oeuvre de Tournier témoigne du fait qu'elle s'interroge sur la responsabilité de l'homme que Moïse rattache à l'essence de la morale prophétique : « C'est la justice, oui la justice que tu dois chercher ». Dans ce sens, l'oeuvre de Tournier - marquée par une énonciation largement prophétique et faisant toujours place à la voix de l'Autre - nous permet de penser la place de l'écrivain dans la culture contemporaine en rapport avec la Loi. Dans cet article, nous tenterons de dégager le discours moral du roman qui laisse entendre la voix des prophètes et de problématiser l'appropriation que Tournier fait de leur langage, de leur mission et de leur morale (ramener le peuple à la justice). Se manifeste ainsi le désir du roman qui, en subvertissant, pervertissant, détournant... bref transposant le message prophétique, pose les linéaments d'une morale qui se fraie une voie dans l'ambiguïté. 


\title{
Transposition de la morale des prophètes dans la trilogie de Michel Tournier
}

\author{
Nancy SAAD" \\ Littérature française \\ Université de Balamand (Liban)
}

Pour qui lit le dernier roman de Michel Tournier Éléazar ou la source et le buisson, inspiré par le livre Moïse d'André Chouraqui ${ }^{1}$, un trait singulier ne devrait pas manquer de le frapper: l'histoire est déchiffrée selon un modèle biblique. Dans ce roman écrit au second degré, Tournier revisite l'exode biblique mettant en scène le sauveur du peuple des Hébreux, Moïse, auquel Dieu apportera délivrance et soutien pour faire sortir son peuple d'Égypte. L'hypertexte suit Éléazar dans sa quête morale, et explicite amplement son identification au grand prophète juif. Fidèle au récit biblique, Tournier l'est — partiellement —, ne serait-ce que pour permettre à son texte de construire un discours du roman qui expose implicitement des positions ambivalentes face à la religion et à la morale. En effet, le rapprochement établi entre l'homme juste, le premier grand initiateur de la religion d'Israël, et le pasteur assassin — dont le crime demeure sans punition - moralement dégradé, parce qu'il fut incapable de remplir son rôle de juge et de chef spirituel avec intégrité et honneur, a des implications morales qui font osciller le discours du roman entre justice et injustice. Il en résulte une ambiguité morale que balisent les renvois récurrents à la Bible et le déploiement d'une énonciation largement prophétique. En reprenant et en développant dans ce roman ${ }^{2}$ les procédés de détournement qui

\footnotetext{
* Nancy Saad est professeure vacataire de littérature française à l'Université Libanaise et à l'Université de Balamand au Liban. Sa thèse de doctorat (2014) est intitulée «Poétique de l'ambivalence. Réécritures et vocation prophétique dans l'œuvre romanesque de Michel Tournier».

1. Dans Éléazar ou la source et le buisson, à la page 108, Tournier souligne dans une note infrapaginale les emprunts faits "au livre fondamental d'André Chouraqui Moïse» qui «a inspiré le [...] roman».

2. Par souci de commodité, les titres des romans et essais de Tournier seront abrégés. Veuillez consulter la liste des abréviations figurant à l'annexe.
} 
ont servi de noyau allégorique à son projet d'écriture, Tournier semble mettre au point un désir de perversion et de subversion traversant l'intégralité de son œuvre et caractérisant particulièrement les trois premiers romans ${ }^{3}$ qui feront l'objet de cet article.

La Bible 4 , que Tournier considère comme étant «le livre le plus important de [sa] bibliothèque » (Lee Luk 2003, 207), lui fournit non seulement des scénarios, des personnages et des lieux mais aussi des registres et une tonalité prophétiques et apocalyptiques toujours indépassés. Sa présence dans l'œuvre témoigne du fait qu'elle s'interroge sur la responsabilité de l'homme que Moïse rattache à l'essence de la morale prophétique: "C'est la justice, oui la justice que tu dois chercher» (Dt 16,20). Cette injonction qui fonctionne comme un leitmotiv chez les prophètes bibliques, notamment Amos et Michée, «a pour effet de responsabiliser l'être humain, toujours capable de choisir entre le bien et le mal» (Cerbelaud 2001, 105). Dans ce sens, l'œuvre de Tournier — faisant toujours place à la voix de l'Autre - permet de penser la place de l'écrivain dans la culture contemporaine en rapport avec la Loi. Cet article propose d'éclairer, à travers l'expérience de l'injustice sociale dans la vie de certains personnages, le traitement du registre moral dans la trilogie tournierienne.

Comment la préséance d'un tel discours moral est-elle problématisée dans l'œuvre littéraire où viennent interférer les différents discours sociopolitiques de la modernité? Exégètes, philosophes et penseurs s'accordent sur un point: le prophétisme "manifeste [...] un état de guerre et de désordre [...]. Au moment des crises, il déborde comme un torrent » et

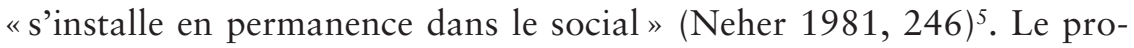
phète, homme d'esprit et de parole perçoit la catastrophe imminente, annonce l'avènement d'un reste, et actualise, dans un optimisme opiniâtre,

3. La trilogie tournierienne se compose des trois premiers romans: Vendredi ou les limbes du Pacifique, Le Roi des Aulnes et Les Météores.

4. Tournier réfère toujours à la Bible commentée en vingt volumes, héritée de son grand-oncle Gustave Fournier.

5. Dans son étude sur le rôle social du prophète biblique, Neher affirme que «le prophète apparaît là où le lévite est en défaut. Lorsque le tissu social est anémié par l'absence du lévitisme, le prophétisme s'y introduit comme un virus. De brusques réactions actualisent alors le lévitisme et le restituent. Par le lévitisme, la berith est rendue possible; par le prophétisme, elle devient désirable. Par l'existence des lévites, la mission lévitique d'Israël est enseignée; par la virulence des prophètes, elle est souhaitée. Le lévitisme met la berith [alliance] à la portée de chaque intelligence; le prophétisme, à la portée de chaque conscience. Le lévitisme fait connaître; le prophétisme, comprendre». 
la délivrance prochaine. Autrement dit, dans la pensée biblique, Dieu appelle le prophète pour rappeler au peuple d'Israël la loi (la Torah) qu'il est en train d'oublier (Draï 1993, 12). Tel est donc l'enjeu de sa vocation prophétique: «Fils d'homme, je t'envoie vers les enfants d'Israël.» (Ez 2,3). Contrairement aux faux-prophètes (Mt 24,23-24) ou prophètes de mensonges qui, selon la tradition biblique, sont «les responsables de l'égarement du peuple $[. .$.$] que Dieu n'a pas envoyés », ceux qui « loin de dégager$ la solution de la crise, [l'enveniment] et [rendent] l'inévitable dénouement encore plus douloureux» (Draï 1993, 284), contrairement à ceux, donc, qui «[hypothèquent] l'avenir en abandonnant le présent à la stagnation, au marasme » (284), les vrais prophètes «ramènent le peuple à la justice » (285), l'incitent à la droiture et à la conversion, transmettent la parole de Dieu et s'efforcent de réduire l'écart qui existe entre cette parole et sa conduite. Ils sont bien les annonciateurs «du joug» (282), ils exigent «son acceptation et [n'assignent] pour fin de l'épreuve, la restauration du royaume et le rétablissement du culte qu'au jour décidé par Dieu, jour imprédictible par l'homme» (282).

Le discours romanesque tournierien, quant à lui, noué autour d'un propos ambivalent sur la justice sociale, permet de sonder le souvenir d'une voix morale (judéo-chrétienne) enfouie et parasitée. Or il importe de souligner que dans cette œuvre littéraire, la volonté de Dieu - si elle existe — n'est pas explicitement formulée. De même, Dieu ne figure pas en tant qu'invocateur (comme dans la Bible), encore moins en tant que législateur d'une loi morale et aucun des personnages n'est prophète au sens strict. Si Dieu s'adresse aux personnages de Tournier c'est à travers les Écritures et les intermédiaires. Dans cette perspective, nous parlerons — empruntant la formulation à Raphaël Draï — d'une

réémergence du prophétique mais paradoxalement dans un espace circonscrit et équivoque: celui de la littérature, autrement dit d'une intervention pseudo-prophétique parce que, si elle met en cause le pouvoir et l'injustice [entre autres], elle le fait dans des limites et des conditions contrôlées par le pouvoir. (Draï 1993, 107)

L'étude de cette «réémergence du prophétique» dans l'œuvre de Tournier n'est en fait soutenable qu'à la lumière d'une poétique de l'ambivalence, elle-même tributaire d'une auscultation de l'homme dans sa conduite morale: sa perversion, sa souffrance, sa perdition, sa conversion et sa rédemption. Tournier réfléchit sur les mœurs et la condition humaine, mais affirme avec ses contemporains que «la présence d'une thèse exposée 
explicitement et s'imposant sans ambiguité, nuit gravement à la valeur de l'œuvre ", qu'elle la condamne à la médiocrité "relevant de l'édification religieuse ou du réalisme socialiste" $(\mathrm{VV}, 16)$. Disposant d'une matière bouillonnante et bouleversante, il organise volontairement dans ses romans à l'écriture classique des espaces d'indétermination et appelle surtout une méditation personnelle, en quelque sorte forcée par les dispositifs qui rendent le statut des actes et des actions du récit ambigu. Le choix de la discontinuité dans son œuvre, en privilégiant l'ambivalence, nous incite à réfléchir sans relâche sur «la relativité de nos mœurs et de la morale en usage par un phénomène d'inversion généralisée qui nous rejette sans trêve ni répit à l'opposé de nos provisoires certitudes" (Magnan 1996, 16), et atteste de l'infinie diversité des comportements humains et de la complexité d'un réel désormais sans cohérence ni signification assurées.

Désignant la coexistence de valeurs qui s'opposent, la notion d'ambivalence requiert d'être appréhendée non seulement à partir de la dialectique de la justice et de l'injustice, mais surtout en fonction des effets de sens qui reposent souvent sur un retournement (détournement) des valeurs produisant la rencontre de situations à la fois salutaires et destructrices. Un commentaire de Jean-Marie Magnan explicite d'une façon générale la dimension essentielle de cette ambivalence morale: "Charnière d'un prompt retournement, les écrits de Tournier nous rejettent de l'une à l'autre des maximes et des règles établies tandis que les extrêmes nous touchent par un phénomène d'inversion généralisée qui nous fait courir sans trêve ni répit à l'opposé.» (Magnan 1996, 16). En effet, le fait d'envisager un possible retournement de l'injustice en justice et inversement, fréquent chez Tournier, participe pleinement de son travail. Dans sa trilogie, qui amorce son parcours littéraire, il semble mettre en place une justice se heurtant à la vie des personnages exclus, marginalisés, pervers, bref voués à la déchéance aussi bien physique que morale. Mais bien loin de les faire sombrer dans la perdition — « recouvert par le manteau de la fable, le plus sinistré a droit au salut » écrit Magnan $(1996,16)$ - Tournier leur propose des issues en quelque sorte rédemptrices, bien qu'ambivalentes, qui permettent de dégager un "discours du roman", alimenté par un sens théologique et spirituel hérité des Écritures.

\section{La subversion de la justice coloniale}

Aventure spirituelle sur une île déserte, Vendredi ou les Limbes $d u$ Pacifique, met en scène un univers colonialiste à échelle réduite qui doit 
servir de révélateur de la fragilité de la condition humaine — et ce, essentiellement sur un versant masculin - et tracer l'évolution morale du personnage principal dominé et modelé par un système de valeurs injuste. Dès le paysage apocalyptique liminaire, l'angoisse et la peur sont aux aguets; elles provoquent chez Robinson, «le reste» sauvé du navire La Virginie, un sentiment insoutenable au goût très âpre: l'injustice. Dans les premiers chapitres du roman des références récurrentes au Déluge inscrivent Robinson, qui vit sans Autrui, dans la violence et la corruption de la génération condamnée. La première inondation catastrophique, relatée dans le prologue, expulse Robinson du fond du navire, du cœur de la civilisation humaine corrompue, et l'emporte sur une île déserte. Dans sa "peur de perdre l'esprit » (VLP, 23), il décide d'entreprendre la construction d' « un bateau de tonnage suffisant pour rallier la côte chilienne occidentale» (VLP, 23), d'une "embarcation qu'il baptisa par anticipation l'Évasion" (VLP, 25). Pour ce faire, il procède à l'évacuation de l'épave «suspendu[e] sur un roc» et décide "d'établir son chantier» afin de précipiter l'avènement du «salut» (VLP, 26). Le mot «salut» qui revient en leitmotiv ( «baie du salut; navire du salut; le salut ») et qui est, dans la logique coloniale de l'homme blanc, strictement lié à la mer, à l'expédition, au voyage, bref à l'évasion, éveille des échos bibliques, explicitement formulés par le narrateur omniscient :

Avant de se mettre au travail, Robinson lut à haute voix quelques pages de la Bible [...]. Ce jour-là, il crut trouver dans le chapitre IV [VI] de la Genèse - celui qui relate le Déluge et la construction de l'arche par Noé - une allusion évidente au navire de salut qui allait sortir de ses mains. (VLP, 26-27)

La référence explicite à l'Arche de Noé provoque dans l'esprit du lecteur (mais surtout du personnage chez qui se remarquent très vite les effets de l'identification) un rapprochement rapide avec le prophète ${ }^{6}$, rapprochement balisé jusqu'alors par l'activité de la construction du navire ainsi que par sa portée prophétiquement rédemptrice. Comparant ce navire «qui allait sortir de ses mains" (VLP, 27) à celui, salutaire, sorti des mains de Noé sous ordonnance divine, Robinson suggère (ou plutôt espère) un sort analogue à celui du patriarche.

6. Dans la Bible, Noé est considéré comme un «homme juste », le seul qui «marchait avec Dieu » (Gn 6,12). Pour la Aggadah, Noé représente, bien qu'avec réticence, « un prophète, un homme de justice, un maître de sa génération et un héros persécuté pour ses blâmes et son honnêteté. " Dictionnaire encyclopédique du judaïsme, "Noé». 
Cependant, un des éléments clés du récit biblique est l'ordre temporel des évènements. Pour le Dieu de Noé, la construction de l'arche n'est envisageable qu'avant le déluge. L'injonction «fais-toi une arche» n'acquiert son sens que dans le prolongement incitatif de la narration: "Entre dans l'arche, toi et ta maison [...] car dans sept jours, je vais faire pleuvoir sur la terre pendant quarante jours et quarante nuits, j'effacerai de la surface du sol tous les êtres que j'ai faits. » (Gn 7,1-4). La construction de l'arche précède alors le déluge. Certains exégètes voient dans cette succession, non seulement une protection sélective mais aussi une chance de rémission collective. Un midrach ${ }^{7}$ rapporte que Dieu «demande à Noah de planter des arbres dont le bois devait servir à la construction de l'arche. Ainsi ces hommes, en voyant Noah procéder à chacun des préparatifs devaient-ils s'amender et revenir à de meilleurs sentiments» (Sabbah 1994, 50). Ce midrash, selon Sabbah, tient à "laver Dieu de l'accusation de frapper par surprise la génération du déluge. Il use de patience et de longanimité, en donnant répit de cent vingt ans» Sabbah (1994, 50). L'indulgence divine fait donc qu'il ne souhaite à aucun moment la mort de l'homme pécheur mais sa conversion pour la vie.

L'inversion temporelle que Robinson insinue dans ses pensées — «le Déluge et la construction de l'arche par Noé» - est bel et bien un procédé de détournement susceptible d'engendrer la confusion sémantique au niveau de la réception de l'intertexte. Robinson, en évoquant en premier lieu le déluge, réfère sans aucun doute au naufrage du navire et à la mort de ses compatriotes. Un évènement tragique subitement enclenché, qui emporte par surprise, qui annihile sans pitié. La construction du navire n'est alors qu'une suite humainement logique à la fatalité climatique, dans l'espoir d'échapper à l'isolement et à la mort. La manifestation cosmique

7. «Le midrash Tanhouma s'exprime ainsi: Rav Houna dit au nom de rabbi Yossi: pendant cent vingt ans le Saint béni soit-Il adressait ses avertissements à la génération du déluge pour l'inciter au repentir. Devant son refus, il recommande à Noah: "Faistoi une arche de bois de gofer". S'étant repenti, Noah se mit à planter des cèdres. Et lorsqu'on lui demande: "Ces cèdres pourquoi les plantes-tu? ” Le Saint béni soit-Il s'apprête, répond-il, à détruire le monde par le déluge et me demande de construire une arche dans laquelle je me réfugierai avec ma famille. Tous se mirent à rire de ses paroles. Le voyant irriguer les cèdres plantés, ils lui demandent de nouveau "Que fais-tu? ". Faisant toujours la même réponse, ils ne lui épargnent pas leurs moqueries. Après qu'il eut coupé et taillé les cèdres, ils se renseignent de nouveau sur les intentions [divines]. Noah les en informe, mais les admoneste. Devant leur refus de se repentir [le Saint béni soit-Il] les frappe de déluge tel qu'il est dit: "Dieu effaça toutes les créatures qui étaient sur la face de la terre”. Sabbah $(1994,51)$. 
occasionne alors une réaction ou plutôt une action - discontinue et sans issue - de la part du héros : construire l'arche du salut, non pour s'y réfugier lors du déluge comme dans la Bible, mais pour fuir la terre ferme.

Néanmoins, dans la suite, nous dégageons une insistance de la référence au Déluge qui survient et suspend la construction du navire:

Des brusques averses et des traînées blanches à l'horizon annoncèrent un changement de temps [...] Bientôt un couvercle de nuages parfaitement homogènes s'appesantit d'un horizon à l'autre, et les premières gouttes mitraillèrent la coque de l'Évasion [...] Puis [Robinson] urina, trouvant plaisant d'ajouter sa modeste part au déluge qui noyait tout autour de lui. (VLP, 29)

Le motif du déluge dans l'esprit de Robinson complexifie la structure de l'hypertexte, la dédouble, et fait du personnage un interprète des signes désireux de rejoindre, par son expérience du naufrage, le patriarche de l'humanité. La première référence au déluge, cause du naufrage, nous l'avons souligné, est cosmique. Les agitateurs sont bien connus: "le vent» (VLP, 7) et «la pluie noire» (VLP, 15). L'allusion qui consiste à "ajouter au déluge» décrit sur un mode ironique l'état du personnage qui «collabore » à l'interprétation de la situation mais aussi l'état du texte lui-même qui se construit sur cette référence. De victime châtiée subissant les conséquences pénibles d'un déluge cosmique, Robinson devient, à un degré minuscule, démiurge. Cette collaboration semble lui procurer un certain plaisir: "Il se sentait soudain en vacances, et un accès de gaieté lui fit esquisser un pas de danse» (VLP, 29). Entre le naufrage et le déluge d'averses, la construction du navire s'entreprend par intermittence, scandant ainsi les hésitations du personnage.

De toute évidence, le naufrage initial, la construction de l'arche et les récurrences diluviennes ouvrent le texte à une interprétation prophétique et messianique placée sous le signe de l'arc-en-ciel et de l'auréole:

Alors, tandis que le grondement de la pluie redoublait sur les feuillages et que tout semblait vouloir se dissoudre dans la nuée vaporeuse qui montait du sol, il vit se former à l'horizon un arc-en-ciel plus vaste et plus coruscant que la nature seule n'en peut créer. Plus qu'un arc-en-ciel, c'était comme une auréole presque parfaite, dont seul le segment inférieur disparaissait dans les flots, et qui étalait les sept couleurs du spectre avec une admirable vivacité. (VLP, 31)

Dans ces quelques lignes, une dynamique eschatologique prévaut: l'abattement diluvien est suivi d'une révélation divine, d'une alliance et 
d'une recherche du sens moral, d'une loi (chiffre sept). Puisant ses éléments dans le texte biblique et les projetant dans la modernité de son récit, Tournier superpose à Noé, homme juste, «croyant silencieux qui obéit » (Drewermann 1995, 293), un Robinson moderne dont il mettra en scène la pureté dépravante et l'injustice. En effet, durant son séjour sur l'île, Robinson ne manquera nullement de déroger à la majorité des sept lois noachides ${ }^{8}$ auxquelles réfèreraient dans le texte de Tournier les sept couleurs de l'arc-en-ciel: l'assassinat non perpétré mais tant désiré de Vendredi, les unions illicites avec Speranza, la profanation de la parole divine qui incite à la droiture et à la justice. Contrairement à la conduite du patriarche, celle de Robinson, imbibée d'orgueil et de haine, atteint un degré élevé d'iniquité surtout avec l'apparition de Vendredi. Dans son log-book, le colon, parlant de lui-même, ébauche les traits d'un humain malfaisant foncièrement jaloux et destructeur:

Je me suis demandé pour la première fois si je n'avais pas gravement péché contre la charité en cherchant par tous les moyens à soumettre Vendredi à la loi de l'île administrée, marquant par-là qu'à mon petit frère de couleur, je préférais la terre modelée par mes mains. (VLP, 168)

En l'assujettissant aux règlements de l'île, il institutionnalise la violence et empêche la coexistence pacifique. C'est ainsi qu'abreuvé au fanatisme, Robinson se détourne des lois divines, et s'enfonce dans l'idolâtrie et l'adoration d'autres dieux «idéologiques".

La vision du personnage ne se limite pas à la représentation vétérotestamentaire de Noé mais s'ouvre sur sa réception chrétienne. Dans l'Évangile, l'histoire de Noé alimente les paraboles de Jésus sur la vigilance qu'il faut toujours garder de l'imminence des temps apocalyptiques; elle préfigure «l'avènement du Fils de l'Homme ( Lc 17,26), le «futur jour de jugement et de destruction des hommes impies» (2 P 2,5):

Ce qui arriva du temps de Noé arrivera de même à l'avènement du Fils de l'homme. Car, dans les jours qui précédèrent le déluge, les hommes mangeaient et buvaient, se mariaient et mariaient leurs enfants, jusqu'au jour où Noé entra dans l'arche; et ils ne se doutèrent de rien, jusqu'à ce que le déluge vînt et les emportât tous: il en sera de même à l'avènement du Fils de l'homme. (Mt 24, 36-39)

8. Selon le Dictionnaire encyclopédique du judaïsme, les «Lois noachides » sont sept lois imposées à Noé: justice civile; interdiction du blasphème; rejet de l'idolâtrie; interdiction de l'inceste; interdiction du meurtre; interdiction du vol; interdiction de manger la chair découpée d'un animal vivant. 
Dans le texte de Tournier, il nous est permis de voir, à travers des indicateurs temporels tels que «soudaine» (VLP, 7), «brusques» (VLP, 29) et «soudainement» (VLP, 31), ce surgissement imprévisible que rappellent les passages évangéliques évoquant l'arrivée impromptue du jugement qui prendra chacun pour la vie ou pour la mort ( "Où sera le corps, c'est là que se rassemblent les vautours » (Lc 17, 37)). Dans le roman, les vautours sont déjà au-dessus de Robinson ( «c'était bien assez des vautours qu'il avait affriandés par une première imprudence et qui depuis le surveillaient sans relâche » (VLP, 28)), une présence qui appelle sans cesse à la vigilance: «Prenez garde, restez éveillés, car vous ne savez quand ce sera le moment.» (Mc 13, 33).

Dans un temps ultérieur, la théologie eschatologique verra dans l'Arche l'image de l'Église: Noé préfigure Jésus, les deux assumant une fonction salvatrice. Marc Bochet (2011, 11-12) souligne que «Tertullien est le premier à faire du thème de l'Arche un symbole explicite de l'Église, lors de l'épisode de la tempête apaisée par le Christ devant un saint Pierre médusé: “Au reste la barque préfigurait l’Église qui, sur la mer du monde, est secouée par les vagues des persécutions" " (De baptismo, XII, 8).

Dans cette perspective, Saint Augustin postule que «par le mystère du déluge était prédite l'Église à venir que le Christ, son roi et son Dieu, par le mystère de sa croix, a maintenu à l'abri du naufrage de ce monde ${ }^{9}$ ». Ce symbolisme ecclésial du navire, Tournier l'instaure, après l'avoir perverti, à travers l'image admirable de la Virginie suspendue "sur un roc" (VLP, 20). Or, au lieu d'impliquer l'idée d'une apothéose ecclésiale, l'allusion à la métaphore de Simon-Pierre — «Tu es pierre, et sur cette pierre je bâtirai mon Église, et la puissance de la mort n'aura pas de force contre elle» (Mt 16,13-20) — injecte dans la narration une détérioration du navire-Église, secoué violemment par l'eau diluvienne.

Dans Le Vent Paraclet, Tournier insiste sur le rôle initiateur que l'Église doit assumer auprès des croyants. Malheureusement, constate l'écrivain, elle ne cesse de se dérober à ce rôle:

Mais il y a l'autre face de l'institution cléricale, hideuse, hypocrite, haineuse. Sa puissance temporelle lui ayant été retirée, l'Église s'est placée comme bonniche au service de la bourgeoisie la plus crasseusement conservatrice dont elle a épousé dès lors ardemment les intérêts et les idées. C'était toujours certes dans les Évangiles qu'elle puisait son enseignement, mais en choisissant la parole des Pharisiens, non celle de Jésus, c'est-à-dire en prê-

9. Augustin d'Hippone, première Catéchèse, 19, 23. 
chant le respect des hiérarchies sociales, de l'argent, des puissants, et la haine du sexe. (VP, 63-64)

Cette Église que Tournier juge pudibonde, «hideuse, hypocrite, haineuse » (VP, 63) semble être représentée par le navire anglais Virginie qui subit la pire des destructions. Le récit biblique a incité les docteurs chrétiens à voir «dans l'arche de Noé une préfiguration de l'Église hors de laquelle (comme à l'extérieur de l'arche pendant le déluge) il n'y a pas de salut, et dans le déluge lui-même une figure du baptême » (Emmanuel 1971, 135-136). À considérer ce commentaire dans le contexte fictionnel, nous sommes invitée à voir dans la Virginie l'incarnation de l'Église coloniale de Robinson dans laquelle il n'y a point de salut. Le déluge, sans nier sa puissance dévastatrice vétérotestamentaire, est par excellence une métaphore baptismale: «Il s'attarda un moment à regarder l'eau tiède ruisseler sur son corps couvert de croûtes de terre et de crasse qui fondaient en petites rigoles boueuses. » (VLP, 29). Il débarrasse le catéchumène des vêtements de la civilisation colonialiste outrageusement injuste et hypocritement pudibonde, le décrasse, le purifie.

Enfin, il y a fort à parier que les éléments constitutifs du texte charrient explicitement l'identification de Robinson à Noé. Mais la convocation de ce prophète constitue pour le colon une identification provisoire, une étape dans son cheminement, qui, à un certain moment, le conduit à une impasse:

Il convient d'ajouter qu'il avait été fortement obnubilé aussi par l'exemple de l'arche de Noé qui était devenue pour lui comme l'archétype de l'Évasion. Construite en pleine terre, loin de tout rivage, l'arche avait attendu que l'eau vînt à elle, tombant du ciel ou accourant du haut des montagnes. (VLP, 36)

Le verbe «obnubiler» remplit ici une fonction double. La première, narrative, qui assure une prise de conscience du personnage, qui lui permet relativement de dévoiler le sens de la catastrophe et, par conséquent, d'invalider l'identification au prophète biblique. La seconde fonction permet au lecteur-interprète de déchiffrer le travail intertextuel de l'auteur. Si Tournier emprunte ses signifiants à la Bible, c'est pour énoncer, du mieux qu'il peut, l'apocalypse des textes antiques dans les mots d'aujourd'hui. S'il convoque Noé dans les premiers chapitres, c'est moins pour construire une identification non méritée du colon injuste au prophète juste que pour dire la fin des expéditions coloniales et le commencement d'un long cheminement - qui passe par la figure provisoire du patriarche - spirituel et moral dans l'île. À la série de termes et expressions disséminés dans le roman et référant à la fin diluvienne, se superpose une autre signalant le 
commencement et le renouvellement: "Une ère nouvelle débutait pour lui - ou plus précisément, c'était sa vraie vie dans l'île qui commençait après des défaillances dont il avait honte et qu'il s'efforçait d'oublier.» (VLP, 45).

Enfin, l'inversion que Tournier fait subir à son personnage - et implicitement à l'Église et à ses représentants spirituels et séculiers - a valeur de subversion morale: par la destruction du navire anglais, un bouleversement des crédos ecclésiaux et patriarcaux est fomenté. Les discours religieux et sociopolitiques convoqués contribuent à la construction d'un sens moral qui affirme l'opposition à l'archaïsme, à l'intolérance et aux abus des institutions périmées des siècles précédents. Le nouveau commencement que Tournier propose à son personnage est une incitation au changement bénéfique qui consacre le renversement d'une hiérarchie sociale malsaine, fondée sur l'iniquité et la suprématie raciale, et qui prélude à la fondation d'un nouveau système de valeurs adapté à la modernité des temps nouveaux et meilleurs.

\section{Abel et Tiffauges: le juste et l'injuste}

Il existe, dans Le Roi des Aulnes, une voix capable de transporter le personnage principal hors de ses repères spatio-temporels, une voix, sinon "prophétique ", du moins habitée par une réalité qui le dépasse et qui fait osciller le discours du roman entre moralisme et immoralisme. Parce que cette voix avertisse Tiffauges, par des paroles millénaires, de la mise en marche de la "Grande Tribulation» (RDA, 101) et de la prise en charge de son «modeste destin» par «le Destin» (RDA, 167), qu'elle s'exprime par des emprunts et des résonances bibliques où l'on retrouve les accents apocalyptiques, elle inscrit dans le roman une lignée sacrée, celle des prophètes - messagers justiciers - s'achevant en Jésus, le verbe qui s'est fait chair.

Dans ses «Écrits sinistres », Tiffauges retrouve des souvenirs immémoriaux qui lui permettent de porter un regard critique sur ses postulats prophétiques, encore plus, de développer son «pouvoir surnaturel» (RDA, 13), lequel se déploie dans la réalisation de ses présomptions: "La nuit fut affreuse, traversée de cauchemars et de longues heures d'insomnie. Une image s'imposait à moi avec une insistance obsédante, celle des flammes qui s'élevaient soudain autour de moi dans la chapelle.» (RDA, 89).

Tout commence avec ce rêve prémonitoire - expression d'un désir - que Tiffauges fait après sa fugue du collège et dans lequel il envisage sa disparition, afin d'éviter de justesse le châtiment que les pères s'apprêtent 
à lui infliger, pour punir une infraction qu'il aurait commise avec Clément dans la chapelle byzantine. La manifestation du feu, élément destructeur et purificateur dans l'œuvre en général et dans Le Roi des Aulnes en particulier, est la clef de voûte de l'énonciation eschatologique, du jugement dernier, qui permet de maintenir, par ses caractéristiques intrinsèques, la cohésion des multiples éléments narratifs l'entourant. Commentant son rêve, Tiffauges insiste sur la fonction ambivalente, punitive et libératrice, du feu: "flammes de l'enfer certes, mais aussi flammes libératrices, car si Saint-Christophe brûlait, si le monde entier brûlait, mon malheur serait lui aussi englouti» (RDA, 87). Ce rêve annonçant une catastrophe imminente suggère "en même temps quelque chose de jubilatoire à voir le monde s'anéantir» (Poirier 2005, 75). Une satisfaction vengeresse que Tiffauges réclame à cor et à cri dès qu'il prend connaissance de l'incendie de SaintChristophe:

Ces hommes stupides qui s'apprêtaient à m'écraser pour une peccadille dont j'étais de surcroît innocent - ne reconnaîtraient jamais, quand bien même je leur hurlerais la vérité en pleine face, la part que j'avais dans le châtiment qui venait de frapper Saint-Christophe. (RDA, 89)

Faisant partie d'un réseau terminologique référant au jugement dernier, les flammes deviennent une métaphore du châtiment. Comme le remarque Jacques Poirier dans "L'apocalypse dans Le Roi des Aulnes ", Abel vit "dans la certitude qu'un processus [d'anéantissement] est en cours» (Poirier 2005, 77). Il est à l'affût des signes dont le déchiffrement lui donne l'impression «de vivre les derniers jours d'un monde qui ne sait pas combien le châtiment est proche» (Poirier 2005, 77):

Et moi, caché parmi les assis, faux sédentaire, faux bien-pensant, je ne bouge pas certes, mais j'entretiens et je répare cet instrument par excellence de la migration, l'automobile. Et je prends patience parce que je sais qu'un jour viendra où le ciel, lassé des crimes des sédentaires, fera pleuvoir le feu sur leurs têtes. Ils seront alors, comme Caïn, jetés pêle-mêle sur les routes, fuyant éperdument leurs villes maudites et la terre qui se refuse à les nourrir. Et moi, Abel, seul souriant et comblé, je déploierai les grandes ailes que je tenais cachées sous ma défroque de garagiste, et frappant du pied leurs crânes enténébrés, je m'envolerai dans les étoiles. (RDA, 51)

La vocation «prophétique» du héros tournierien «Abel» est précisée à partir de son jumelage à la première victime dans l'histoire de l'humanité: il espère présider à la victoire de sa lignée et menace de perdition les caïnites «endurcies contre leurs frères nomades» (RDA, 51) et accusés de 
crimes et d'abominations. Il patiente et «attend donc l'heure du jugement où justice sera enfin rendue» (Poirier 2005, 77). L'opposition entre Abel et Caïn, dans laquelle se rangent toutes les formes de l'expérience existentielle de l'histoire, permet à Tournier de construire l'ambivalence tout en condensant en Abel Tiffauges deux tendances contradictoires. Cette coexistence, Armand Abécassis la rappelle tout en posant qu'«il ne fait pas de doute que chacun de nous est Caïn et Hével [...] Chaque homme est à la fois victime et coupable» (Eisenberg et Abécassis 2004, 642-643). Les propos de Tiffauges ne se limitent pas à la temporalité mythique de l'Écriture, mais ils s'universalisent et s'actualisent afin de dépeindre une situation - conflictuelle - touchant l'humanité en général, situation qui, d'après les dires de ce personnage, «se poursuit de génération en génération depuis l'origine des temps jusqu'à nos jours» (RDA, 51). Dans Le Roi des Aulnes, dès que cette réflexion surgit dans le chapitre des "Écrits sinistres ", les structures morales du récit se mettent en place, avec toutes les conséquences que peut engendrer un tel antagonisme (entre sédentaires et nomades / nazis et gitans) sur le contexte sociopolitique de l'histoire: l'affrontement, la haine, la violence, la criminalité:

Et cette haine n'est pas éteinte, bien loin de là, elle se retrouve dans la règlementation infâme et infamante à laquelle les gitans sont soumis — on les traite comme des repris de justice - et elle s'affiche à l'entrée des villages par les panneaux «Stationnement interdit aux nomades. ( RDA, 50)

Le rapprochement établi entre Caïn, les sédentaires et les nazis touche également les actions ainsi que les séquelles de ces actions. Autrement dit, dans la Bible, Dieu maudit Caïn. Ce dernier est selon Tiffauges «condamné à la pire des peines »: "Maintenant, lui a dit l'Eternel, tu seras maudit de la terre qui a ouvert sa bouche pour recevoir de ta main le sang de ton frère. Quand tu cultiveras la terre, elle ne te donnera plus ses fruits, tu seras errant et fugitif dans la terre. » (Gn 4,11-12). La punition infligée à Caïn insinue dans le discours du roman la possibilité du châtiment pour les meurtres perpétrés par les nazis à l'égard des Gitans. Au dernier chapitre, les représentants du régime nazi connaîtront la destruction et l'errance. De persécuteurs, ils deviennent persécutés. La parole prophétique de Tiffauges prévenant cette fatalité, annonce d'un même coup la victoire de la lignée d'Abel. Inscrite dans le paradigme de l'ambivalence, du nomadisme et de la sédentarité, l'histoire biblique place la quête de Tiffauges dans une optique d'opacité morale. D'un côté, l'identification à Abel de par son origine filiale permet au personnage principal d'accéder aux rêveries de 
l'élévation et de l'ascension. De l'autre côté, on le trouve acculé à la pesanteur de la sédentarité maudite.

L'expérience de l'injustice perturbant la quiétude de son enfance au collège, les incidents qui l'émaillent et surtout la libération consécutive, fournit à Tiffauges, adulte, les arguments nécessaires pour se défendre contre l'injustice, suite à l'affaire Martine, à son accusation «mensongère » de viol. Son cri de condamnation s'élève: "L'école va brûler, comme il y a vingt ans à Beauvais. Mais cette fois, l'incendie sera à la mesure du géant Tiffauges et de la terrible menace qui pesait sur lui.» (RDA, 173). Le ciel ne tarde pas à répondre: "l'école a brûlé encore une fois " (RDA, 177) et "la société sous laquelle Tiffauges avait souffert était balayée avec ses magistrats, ses généraux et ses prélats, ses codes, ses lois et ses décrets » (RDA, 216). Durant cette période troublante d'avant-guerre, le «prophétisme prémonitoire» de Tiffauges s'installe en partie, nous semble-t-il, dans le social. Dans ses aspirations personnelles, on voit poindre une destitution, un revirement d'un état sociopolitique qu'il juge injuste. Comme il est malaisé de croire à la droiture morale du personnage, et de déterminer s'il convient de l'accuser ou de l'innocenter, il serait imprudent de ne pas voir dans sa prétendue quête de justice un renversement vicieux des codes pénaux qui réprimandent sévèrement les crimes organisés, en l'occurrence ses agressions. Traitant de la fonction sociale du prophète, Neher affirme qu'elle

est à la fois de destitution et de restitution. En destituant des valeurs fausses et usurpées, elle introduit dans le monde social un courant de moralité. En restituant les valeurs réelles et écrasées, elle fait accéder les plus humbles au niveau historique de l'existence nationale. (Neher 1981, 248)

La vocation du prophète est alors soumise à deux prédispositions complémentaires: la destitution des valeurs usurpées et la restitution des valeurs réelles, d'une morale ancestrale, étant donné que le social «n'est qu'un épiphénomène du moral» (Neher 1981, 251). L'expérience "prophétique» du personnage se concilie mal avec celle des prophètes bibliques, «hérauts de la justice» (Neher 1981, 251). Si destitution il y a, la restitution serait d'une morale équivoque voire perverse, à l'image de ce géant ogre, en qui germe «un grouillement de perversions larvées» (VP, 122). De plus, dans le cadre du prophétisme biblique, toute réflexion sur le rôle du prophète instille une promesse de promotion: «La virulence prophétique [...] destitue les couronnes, les grands, les autonomies, les privilèges, les abus de l'orgueil; elle restitue l'humble, le simple, l'opprimé, le souf- 
frant. ( Neher 1981, 251). Tant d'espérance pour le personnage-prophète abattu, humilié, ridiculisé et souffrant, en qui nous percevons, tout au long du roman, la fécondation d'une convoitise humaine, d'un souhait obscur de désignation, laquelle sera accompagnée d'un sentiment pervers de joie intense - «Il était porté en avant par un pressentiment grave et joyeux » (RDA, 308); «angoisse voluptueuse » (RDA, 271) — et ravitaillée par une brutalité maniaque:

Il souhaita seulement pouvoir bientôt jeter aux orties l'uniforme qu'on lui tendit [...]. Le jour où il revêtirait une tenue d'une autre sorte, enfin accordée à sa dignité véritable, alors oui il saurait - et tout le monde avec lui — que les temps obscurs étaient révolus. (RDA, 217)

Dignité et effacement des temps obscurs sont à appréhender - avec toutes "les révélations décisives» (RDA, 216) du personnage — dans le mouvement ambivalent du discours moral du roman. D'une part, ils pourraient référer à la récupération moralement rédemptrice du personnage, rendue possible, à la fin du roman, par l'obéissance aveugle à l'enfant Éphraïm. D'autre part, ils sauraient pointer les inversions malignes qui articulent la vie de l'ogre et qui agencent au prix d'inconcevables atrocités, son élévation "à une puissance supérieure, encore jamais atteinte " (RDA, 243), sa promotion ogresque au sein du nazisme.

Pleinement fidèles à l'esprit des discours prophétiques qui prédisent catastrophes et délivrance, fin et commencement, les réflexions de l'ogre retirent à la voix fictionnelle son individualité pour la fondre dans un mode d'expression non plus romanesque mais prophétique. La promotion sociopolitique à l'Est ne renforce en vérité chez l'ogre rêvant orgueilleusement de la survenue fatale de son propre royaume (RDA, 368), qu'une interprétation controuvée de ses visions, qu'une ignorance profonde ou plutôt qu'un détournement opiniâtre de la volonté divine. Les registres eschatologiques ainsi intégrés au roman pour exprimer un rêve tiffaugéen de triomphe, d' "ascension" au sein de la napola, gagnent en ambiguïté ce qu'ils perdent en probité. Alors que le discours des prophètes et de Jésus se révèle aux lecteurs de la Bible dans toute sa limpidité rédemptrice, attribué par une sorte de délire extatique au prédateur des enfants, il se prête au détournement de la morale biblique et à l'instauration d'une morale tiffaugéenne qui rejoint par un étonnant renversement l'immoralité historique des nazis. Cela se donne à voir surtout dans le dernier passage du roman. Malgré les déductions tirées à l'égard de la criminalité du régime totalitaire nazi, le cheminement dernier du personnage est soumis à deux 
forces contradictoires. La première, immanente, participe de son impéritie qui se traduit par la volonté de "s'arrêter, faire demi-tour" (RDA, 496). La seconde, transcendantale, pétrifiante et «irrésistible» (RDA, 496) qui «le poussait aux épaules», le faisait avancer malaisément et malgré lui, afin de le forcer à s'engager éternellement et sans retour dans la voie du salut. L'affrontement final entre mal et bien, injustice terrestre et justice divine, Tournier le situe, vers la fin du roman, grâce à l'apparition de l'enfant juif Éphraïm, comme espace communicationnel d'une vérité morale, un espace de démystification qui assure précisément la transition entre le prophète et l'ogre.

L'horizon moral de l'intervention de l'enfant-prophète apparaît in fine dans un avant-dernier jeu phorique: "Cheval d'Israël, emporte-moi, lui disait-il, montre-moi les arbres, il faut que je surveille le dégel qui annoncera la nuit du 15 de Nissan.» (RDA, 483). En s'y pliant, Tiffauges court le risque d'être vu et dénoncé. Ses craintes se sont avérées bien fondées et justifiées. Un soir, le cheval Tiffauges «se trouve nez-à-nez» (RDA, 483) avec un soldat nazi. "Aveuglé par la peur et la colère " (RDA, 484), il cède à la tentation de le tuer. À l'égard de ce geste meurtrier, la morale tiffaugéenne - essentiellement ogresque, nazie — peut être tenue, dans ses grandes lignes, pour un désir d'extermination radicale. Un désir "sourd" qui arrache à Éphraïm «un cri aigu» (RDA, 484) puis légitime l'emploi de l'impératif, en tant que rapport entre commander ("Béhémoth ne le tue pas [...] ne tue pas, non ne tue pas!») et obéir: "Tiffauges ne doutait pas qu'il abdiquerait désormais de plus en plus devant son protégé. Il s'y résignait le sentant plus habité encore que lui-même par la force du destin.» (RDA, 484). Cette conversion à la morale de l'enfant juif qui dénonce l'injustice et le crime et contient une promesse de délivrance, s'achève dans la "récitation" (RDA, 485) psalmodique — «avait psalmodié" (RDA, 485) - des versets bibliques. Or, cette apothéose morale place Tiffauges dans un état d'ambivalence:

Tiffauges, auquel l'image du Roi des Aulnes couchés dans le secret des roseaux et des marécages s'imposait immédiatement, admirait la certitude de l'enfant dans le triomphe final de son dieu, et il se rapprochait de lui comme d'un foyer ardent, pour profiter du rayonnement de sa foi prophétique. (RDA, 485)

Une insistance mnésique "maléfique» semble interférer avec la voix du prophète. Dans l'esprit pervers de Tiffauges, la superposition de l'image du «Roi des Aulnes» à l'espérance prophétique décèle une incompréhen- 
sion du message divin transmis par Éphraïm. Associée aux ténèbres et à la mort, cette image malveillante aiguise la perversion du personnage et rend son destin encore plus inquiétant. Elle parvient à l'emmurer dans une densité morale quasi impénétrable en le poussant à désobéir aux ordres de l'enfant, à "faire demi-tour» (RDA, 496) dans la terre de sa vocation ogresque, mais surtout à rejoindre le sort du Roi des aulnes qu'incarne parfaitement l'image finale de son ensevelissement dans la boue.

En dépit de cette mort, la posture verticale de sa tête ainsi que la vision de l'étoile renferment un espoir lumineux de salvation grâce à la force irrésistible de l'enfant dont le rôle prophétique est somme toute lisible dans son patronyme. De l'hébreu Ephraath qui désigne le "lieu de la fécondité", Ephrayim que l'on traduit par Éphraïm signifie «doublement fécond ». La convocation de ce personnage, de cette double fécondité vient nous semblet-il remédier à la stérilité de l'ogre; elle véhicule la possibilité d'une naissance humaine, d'une renaissance. Quand Éphraïm pose les fondations de la morale divine qui incite à l'indulgence et au pardon («ne le tue pas»), à l'assistance des faibles, à la foi en un Dieu Puissant ("profiter du rayonnement de sa foi prophétique»), à l'obéissance aveugle à la voix du salut («je vais te prendre par les oreilles, et te guider»(RDA, 494)), il provoque le surgissement du remords et de la culpabilité, éveille le côté humain du personnage et assiste à sa renaissance après un long travail d'enfantement. Bref, il enracine Tiffauges, malgré lui, dans un nouveau langage, celui de la justice, dont la tâche est de laver la perversion existante des dernières adhérences du sens nazi, d'en détacher les scories politiques et immorales.

\section{Une juste, un châtiment: Deborah en ce Jour du Seigneur}

L'ambivalence du discours tiffaugéen aide à comprendre les préoccupations morales de Jean et de Paul dans Les Météores, notamment dans le chapitre «L'île des Lotophages " où surgissent corrélativement les deux récits bibliques, la Création et le Déluge, et auxquels correspondent simultanément les registres de destruction et de renouvellement. Dans son récit de la vie de Ralph et Deborah, Jean les compare à Adam et Ève. Venus «les premiers» à EL-Kantara, ils entreprennent la création de leur Paradis (LM, 465):

La création avait commencé. Elle n'a plus cessé depuis, car cette maison, ce jardin à l'opposé du désert immobile et éternel qui les cerne - tiennent registre du temps, à leur manière, gardant trace de tout ce qui arrive et part, des croissances, résorptions, mues, déclins et reverdies qu'ils traversent. (LM, 466) 
Les énoncés du jumeau se présentent comme un cheminement eschatologique: le sens émerge de la bipolarité de la création, oscillant entre déclins («part», «résorptions») et reverdies («mues», «croissances»). Après un long voyage, le retour «impromptu» du couple à l'île annonce une période où la maladie de Déborah s'exacerbe progressivement, pour culminer dans la mort, dans une énonciation agonique et cataclysmique.

Deborah était saisie par une crise de suffocation effrayante. Lorsque nous l'avons débarquée à EL-Kantara dans une tempête de sable, elle agonisait [...]. D'heure en heure une muraille de nuages plombés s'édifiait sur l'horizon. Cependant Deborah gémissait, se tordait les mains, s'accusait comme d'un crime d'avoir si longtemps abandonné son jardin. (LM, 468469)

Sous le regard de Jean, une relation entre l'état de santé de Deborah et l'univers se manifeste dans la juxtaposition de références climatiques et symptomatiques. En l'insérant dans ce réseau sémantique, Jean semble conférer à l'agonie de la «nouvelle Ève» une valeur référentielle et symbolique qu'il ne tarde pas à rattacher à la destruction punitive de son jardin par les eaux du déluge:

Une nuit, la tempête creva enfin à grands fracas sur nos têtes. Cependant que des éclairs nous révélaient une fraction de seconde à quels sévices le jardin était soumis, l'agitation de Deborah devenait angoissante. Sourde à nos supplications, elle voulait sortir à tout prix pour protéger ses créatures, et deux hommes devaient se relayer à son chevet malgré son extrême faiblesse pour la retenir. Le jour se leva sur un spectacle de ruine. Le vent était tombé, mais une pluie dense et régulière crépitait sur les feuilles qui jonchaient le sol. (LM, 470)

De toute évidence, le registre apocalyptique exposera d'une façon très nette l'empreinte punitive de la nature (de Dieu ?) sur Deborah, sans pour autant dénoncer explicitement son œuvre malfaisante. L'énonciateur s'attache à mettre au jour les ressorts catastrophiques du déluge lesquels débouchent sur la mort violente - très symbolique - des deux paradisiers, appelés aussi oiseaux du paradis. Il achève la construction de sa vision paysagère chaotique qui figure le renversement de l'ordre du paradis terrestre de Ralph et Deborah. Référées à la logique biblique de destruction, la mort de Deborah et la détérioration de son jardin se rencontrent autour d'une poétique de la fin, que le frère-pareil de Jean, accentue dans la suite et enchaîne à la notion de châtiment par le lien défini d'une alliance de deux mythes bibliques. 
Le récit de Paul laisse deviner l'importante part du Déluge dans le récit de la fin de Deborah et de son jardin qui n'est plus «qu'un enchevêtrement de troncs abattus, de palmes brisées, de feuilles entassées» (LM, 475). Cette réalité cataclysmique, Paul la rapporte et la confirme: «Deborah disparue, maison et oasis s'effacent de la surface de l'île à une vitesse prodigieuse, effrayante, magique. » (LM, 481). Le choix du verbe «effacer» est très significatif. Emprunté au déluge, notamment à la scène de l'inondation, il est synonyme d'anéantir. L'effacement brutal et surprenant n'est pas dénué de rapport avec la morale biblique, de la justice divine et du châtiment. Ce rapport, Paul s'efforce de l'établir à la fin du chapitre tout en devinant l'opinion d'un personnage secondaire, Tanizaki: «Il [Tanizaki] ne m'a pas caché, en des phrases il est vrai feutrées et allusives, qu'il jugeait sévèrement l'œuvre de Deborah. CEuvre brutale et barbare dont la débâcle à laquelle nous assistons était inscrite dans les origines mêmes. " (LM, 494). Dès lors que s'énonce la pensée de Tanizaki, le récit de Paul prend un tour nettement eschatologique, le «naufrage du couple» (LM, 485) devient assimilable au naufrage biblique, leur attitude à celle de l'humanité idolâtre. En adressant la parole à ce personnage taciturne, Paul tente de spéculer sur la cause d'une telle condamnation:

Deborah s'est acharnée à faire pousser un jardin féerique en plein désert. C'était évidemment faire violence au pays. D'ailleurs le pays se venge avec une hâte stupéfiante, maintenant que la femme aux mains vertes n'est plus là pour défendre son œuvre. Est-ce cette violence que vous réprouvez? (LM, 494)

Paul manque de subtilité et d'intelligence dans son interprétation, Tanazaki n'hésite pas à le lui faire sentir. Toutefois, sur le plan de l'énonciation, le frère déparié a le mérite de fédérer les registres de l'insurrection («s'est acharnée », «faire violence», «violence») et du châtiment («se venge»; " pourquoi le jardin de Deborah est condamné») et de les mettre au service d'un discours moderne qui annonce le "Jour du Seigneur". Quoi qu'il en soit de la vraie cause, l'idée d'un jugement dernier, d'un châtiment pour Déborah et Ralph, les malfaiteurs romanesques, constitue le socle moral sur lequel s'édifie dans ce chapitre le récit de la catastrophe finale, du déluge. Comment, enfin, à travers la condamnation de Déborah, ne pas penser au traitement onomastique pervertissant qui illustre l'ambiguïté extrême du discours moral? En se plaisant à lui accorder le nom de la prophétesse biblique et de la seule femme parmi les juges d'Israël, Tournier forge l'identification possible du personnage romanesque au 
personnage biblique sur un dépouillement substantiellement moral, lequel poussé à son paroxysme, métamorphose la juge en coupable.

\section{Écrire c'est pervertir; écrire c'est subvertir}

Tel est, dégagé dans son complexe développement, le registre de la justice sociale que Tournier emprunte à la Bible, intègre au tissu du roman et traite pour servir la fiction afin d'accentuer un certain enjeu moral. Dès lors, il est devenu manifeste à travers les pages précédentes que la convocation du discours prophétique sur la justice fait simultanément émerger des liens spécifiques et creuser un clivage entre les personnages de Tournier et les prophètes parlant dans la Bible. Ces rapports ambivalents fondent la structure du discours moral et étayent l'hypothèse selon laquelle l'écriture fictionnelle, qui actualise les discours bibliques par l'incarnation des discours sociopolitiques contemporains, témoigne d'une polyphonie, d'une tension — morale - liée à la capacité de provoquer la confusion entre justice et injustice tout en préservant la spécificité de chaque pôle. Cette confusion tient précisément de la coexistence au sein des sujets tournieriens de deux valeurs contradictoires, de l'interférence de deux voix opposées, d'une part la voix de la société corrompue, d'autre part la voix d'une morale ancestrale. L'intrusion de cette morale extrêmement heurtée révèle l'urgence de la libération de manière laborieuse mais irréversible de la voix intérieure de l'homme, un homme nouveau capable du changement bénéfique.

De même qu'elle dit le détournement des valeurs morales et humaines, la trilogie tournierienne met en scène le surgissement d'un retournement rédempteur, d'une conversion salvatrice. L'exploitation textuelle du registre prophétique ainsi que la convocation des références judéo-chrétiennes reflètent le déroulement de tel aboutissement libérateur qui est observable à partir de deux perspectives. La première, subversive, se propose de renverser l'ordre établi et de le remplacer par un nouveau système d'idées et de valeurs. C'est en destituant les idéologies pernicieuses que le personnage ouvre la voie à la restitution des mouvements intérieurs qui conditionnent et permettent le déploiement d'un univers originel hanté par l'obsession eschatologique. La deuxième perspective autorise la saisie des perversions et des transformations de sens subies par le discours moral qui se lisent dans l'inclination des personnages à des conduites considérées comme «déviantes» par rapport aux règles et croyances morales. Ce qui ressort est bien une énonciation prophétique ambivalente porteuse d'une vision morale fusionnant modernité et archaïsme. 


\section{Liste des abréviations}

Vendredi ou les limbes du Pacifique (VLP)

Le Roi des Aulnes (RDA)

Les Météores (LM)

Le vent paraclet $(\mathrm{VP})$

Le vol du vampire (VV)

\section{Références}

Augustin D’Hippone (1991), Première Catéchèse, Paris, Études augustiniennes.

Traduction ecuménique de la Bible (1996) [1887], La Bible. L’Ancien Testament, le Nouveau Testament, Paris, Cerf (Le livre de poche).

Bochet, M. (2011), L’Arche de Noé et la seconde création, Paris, Honoré Champion.

Cerbelaud, D. (2001), "Le thème des "deux voies" dans les premiers écrits chrétiens ", dans S. Trigano, dir., Y a-t-il une morale judéochrétienne?, Paris, Pardès.

Draï, R. (1990), La communication prophétique. Le Dieu caché et sa révélation, Paris, Fayard.

(1993), La communication prophétique. La conscience des prophètes, Paris, Fayard.

Drewermann, E. (1995), Le mal. Structures et permanence, Tome 1, Paris, Desclée de Brouwer.

Emmanuel, (1971), Pour commenter la Genèse, Paris, Payot.

LeE Luk, F. (2003), Michel Tournier et le détournement de l'autobiographie, Dijon, Éditions Universitaires de Dijon.

Neher, A. (1981), Amos. Contribution à l'étude du prophétisme, Paris, Vrin.

Poirier, J. (2005), dir., Tournier, Paris, L’Échelle de Jacob.

SAввAн, D. (1994), La Bible. Le commentaire de la Tora. 1-BérèchitGenèse, Québec, Phidal.

Shemot Rabba (2007), Le Midrash Rabba sur l'Exode, Tome I, Paris, OT.

Tournier, M. (1977), Le vent paraclet, Paris, Gallimard (Folio). (1981), Le vol du vampire, Paris, Mercure de France (Folio essais), 
(1996), Éléazar ou la source et le buisson, Paris, Gallimard. (2005) [1975], Les Météores, Paris, Gallimard (Folio). (2006) [1967], Vendredi ou les limbes du Pacifique, Paris, Gallimard (Folio). 2007 [1970], Le Roi des Aulnes, Paris, Gallimard (Folio).

Eisenberg, J. et AbÉCAssis, A. (2004) [1978-1980], dir., "Moi, le gardien de mon frère ", À Bible ouverte. La Genèse ou le livre de l'homme, Paris, Albin Michel, p. 639-651.

\section{Résumé}

La présence de la Bible dans l'œuvre de Tournier témoigne du fait qu'elle s'interroge sur la responsabilité de l'homme que Moïse rattache à l'essence de la morale prophétique: "C'est la justice, oui la justice que tu dois chercher». Dans ce sens, l'œuvre de Tournier - marquée par une énonciation largement prophétique et faisant toujours place à la voix de l'Autre - nous permet de penser la place de l'écrivain dans la culture contemporaine en rapport avec la Loi. Dans cet article, nous tenterons de dégager le discours moral du roman qui laisse entendre la voix des prophètes et de problématiser l'appropriation que Tournier fait de leur langage, de leur mission et de leur morale (ramener le peuple à la justice). Se manifeste ainsi le désir du roman qui, en subvertissant, pervertissant, détournant... bref transposant le message prophétique, pose les linéaments d'une morale qui se fraie une voie dans l'ambiguité.

\section{Abstract}

The Bible in Tournier's oeuvre proves that it is a questioning of the responsibility of the human being, a responsibility that Moses links to the essence of the prophetic moral: "It is justice that you should seek". In this respect, the work of Tournier, marked by an enuntiation that is highly prophetic and that always prioritize the voice of the other, allows us to think about the role of the author in the contemporary culture in relation with the Law. In this article, we will try to extricate the moral speech of the novel that implies the voice of the prophets, and to study the problematic of how Tournier takes appropriation of their language, their mission and their moral (bringing back the people to justice). Therefore, this shows the desire that the novel holds which, by subverting, perverting, diverting... transposing the prophetic message, installs the lineaments of a morale that opens the road towards ambiguity. 